\title{
Mode hopping and carrier density fluctuations in semiconductor lasers
}

\author{
M. Jadan
}

Applied Physics Department, Faculty of Science, Tafila Technical University

P. O. Box 179, Al-Eys 66110, Tafila, Jordan, e-mail: muhanad_jadan@yahoo.com

Received: 26.09 .2013

\begin{abstract}
We treat the effect of noise on the power and polarization characteristics of semiconductor lasers within the scope of our earlier model which considers polarization switching as a successive process of polarization transformation. We demonstrate that, under particular conditions, one can observe a stepwise transition between the states with the limiting values of polarization degree, though the latter remain statistical in their character. This process requires a certain time for its development, which cannot be regarded as a laser-system parameter.
\end{abstract}

Keywords: semiconductor lasers, polarization switching, fluctuations

PACS: $42.55 . P x$

UDC: $52-626+621.3 .032$

\section{Introduction}

The problem of polarization instability in semiconductor lasers has been attracting attention of researchers for a long time [1]. In the majority of cases, instabilities of this type manifest themselves as a rapid switching of one linear polarization to its orthogonal counterpart at minor variations of such parameters as the injection current, temperature, mechanical stresses, etc. As a rule, the polarization switching (PS) is well observed in vertical cavity surface-emitting semiconductor lasers (VCSEL) [2] when the injection current is changed and has a hysteretic character. On the one hand, these effects may be used for developing various optoelectronic devices [3] while, on the other hand, they are very unwanted in any polarization-sensitive telecommunication systems.

Although numerous studies devoted to the polarization instability problems have been published, some questions concerning the nature and mechanisms of the PS still remain unanswered. To a great extent, this stems from the fact that the effects associated with the PS are interpreted using the models based on a polarization mode method (PMM). Within the scope of the PMM it is assumed that two independent modes with orthogonal linear polarizations formed inside a laser are coupled by some processes described by phenomenological parameters [4, 5], of which experimental determination presents difficulties. For example, to explain a hysteretic character of the PS, researchers often use [5] a difference in the coefficients of self- and cross-saturation of the modes though, as shown by the studies on solid-state lasers [6] where the PMM is widely employed, the cross-saturation parameter cannot be treated as a pure laser-system parameter.

A new phenomenological approach $[7,8]$ has recently been suggested for describing the PS. In the framework of this approach, the process of formation of polarized radiation in a semiconductor laser is governed by orientational anisotropy of the amplification and/or loss factors. In this case the PS represents a transition from one linear polarization to the orthogonal one through a sequence of partially polarized states inside the region of orientational distribution 
for the difference in the amplification and loss factors, and the distribution is close to the isotropic one. Such an approach enables describing qualitatively the main specific features of the PS and finding a fairly simple interpretation, including the conditions for external injection of the polarized optical radiation [8].

Notice that, within the model [7,8], the polarization instability region is determined by a relatively small orientational anisotropy of the difference in the amplification and loss factors rather than by a competition of the two stable polarization states ('polarization modes'). Then the transformation of polarization of the output radiation proceeds at a much slower rate than the formation of its intensity. This is reflected in lowered rate of the PS occurring due to dynamic change in the pump power. In fact, one can state that the PS rate, which corresponds to the range of the injection currents where we observe the transition from one state with a limiting polarization-degree value to the other, is inversely proportional to the rate at which the injection current changes. However, it is well known [9-11] that fluctuations of the laser parameters (those referred to spontaneous emission, carrier density, injection current, etc.) may result in an abrupt change of polarization of the output radiation, thus leading to the effect referred to as a "mode hopping'. Because of this, in this work we consider a possibility for realization of this effect on the basis of our model [7, 8].

\section{Theoretical model}

We use the model $[7,8]$ developed on the basis of a polarization component method (PCM). According to it, a plane wave propagating along the axis $z$ is represented as a superposition of linearly polarized components:

$$
\mathbf{E}(z, t)=\int_{0}^{\pi} \mathbf{e}_{\psi} E(\psi, z, t) \exp [i \varphi(\psi, z, t)] d \psi .
$$

Here the quantities $\mathbf{e}_{\psi}, E(\psi, z, t)$ and $\varphi(\psi, z, t)$ are respectively the unit vector determining the orientation of a linearly polarized component, the amplitude and the phase of a single component, whereas the angle $\psi$ gives orientation of the polarization vector $\mathbf{e}_{\psi}$ with respect to the selected axis in the wave-front plane. According to the PCM, it is believed that, due to the rapid phase relaxation characteristic of semiconductor lasers, some polarization components $E(\psi, z, t)$ are not phase-correlated and their evolutions may be considered separately. Moreover, it has been shown in the work [12] that the polarization distribution of the radiation formed within the cavity of a semiconductor laser is actually homogeneous even for strong inhomogeneities of the intensity distribution. Consequently, we will use dynamic equations averaged over the cavity volume for the intensity of a single polarization component and so we will pass to the rate equations. Then we have

$$
\frac{d I(\psi, t)}{d t}=v\left[\left(g(\psi)-k_{a b s}\right) I(\psi, t)+L_{l u m}(\psi)\right],
$$

where $I(\psi, t)$ denotes the intensity of a single polarization component, $k_{a b s}=\frac{1}{2 L} \operatorname{Ln} \frac{1}{R_{1} R_{2}}-\rho$ the overall loss factor including the cavity mirror losses, $v$ the group velocity, $L$ the cavity length, $R_{1,2}$ the mirror reflection factors, $\rho$ the internal loss factor, $L_{l u m}(\psi)$ the orientational component of the power density for spontaneous emission, and $g(\psi)$ the amplification factor for a single 
polarization component given as [7]

$$
g(\psi)=\frac{g_{0}}{1+\varepsilon I}\left(k_{x} \cos ^{2}(\psi)+k_{y} \cos ^{2}(\psi)\right) .
$$

Here the parameters $k_{m}(m=x, y)$ determine the orientational anisotropy of the amplification factor with reference to the crystallographic axes of the active layer oriented orthogonally to the optic axis of the cavity,

$$
k_{x, y}=a_{x, y}+b_{x, y} J,
$$

$J$ implies the injection current density, $\varepsilon$ the saturation parameter, and $I$ represents the total intensity of the amplified radiation,

$$
I(t)=\int_{0}^{\pi} I(\psi, t) d \psi .
$$

In principle, for a more accurate computation, Eq. (2) should be complemented by a rate equation for the concentration of non-equilibrium carriers, the amplification factor being associated just with this quantity. But this approach would necessitate specifying a particular type of laser with certain recombination parameters, a complete set of which can be rarely found in the literature. The main problem, however, is associated with estimations of the parameter $k_{m}$, involving direct quantum computations which are complex and tedious. Because of this, we use a phenomenological approach to determine the amplification factor $g_{0}$ so that the working range of the injection current densities fall in the region of $1.2-1.5$ of its threshold value $J_{t h}$. In the PS region, the parameters $k_{m}$ are close to unity though $b_{m}$ must be much less than that. The parameters $a_{m}$ and $b_{m}$ are selected to provide the PS region of $0.1-0.2 J_{t h}$. It is necessary to point out that their variations have an effect on both the PS location and its rate however they do not affect the PS as a physical phenomenon.

The noise in semiconductor lasers may be caused by both the spontaneous emission as a random process and the fluctuations of non-equilibrium carrier concentrations over the volume of the active layer, the latter leading to amplification factor fluctuations. However, the present study is aimed at the influence exerted by different fluctuations on the character of the PS rather than on the characteristics of the output radiation. Nevertheless, it is possible to reveal that the statistical character of the spontaneous emission plays no essential role. Taking into account the peculiarities of the model adopted here, we have given consideration to the injection current fluctuations leading to the fluctuations of spontaneous emission intensities and non-equilibrium carrier concentrations, after assuming that these are rather small. Estimations for the heterostructures based on GaAs and InGaAsP for the region where the lasing threshold is in excess by $30-50$ per cent demonstrate that, even in the case when the injection current is varying by about 20 per cent, the changes in the densities of non-equilibrium carriers remain well below 1 per cent.

Eq. (2) has been solved numerically for a sequence of identical-length trapezoid-like injection-current pulses whose maximal amplitude takes the form $J=J_{0}(1+\xi)$, where $\xi$ is the random variable $(\langle\xi\rangle=0)$ obeying the normal distribution, with the relative dispersion $\sigma$ for the distribution $J / J_{0}$. The pulse length has been varied over a wide range under the condition that the quasi-static condition is fulfilled. In principle, an arbitrary pulse shape may be chosen, though the trapezoidal one has the two main advantages. First, it enables minimization of the effects 
associated with the transient processes. Despite the fact that in the work [12] has demonstrated that these processes have a minor effect on the dynamics of polarization formation, it would be better to exclude this factor. Second, the appearance of the plateau results in a monotonic (practically linear - see the results [8]) polarization variation during the pulse, thus making it possible to control, in the simplest fashion, the polarization characteristics of the output radiation. A polarization degree for the output radiation is defined in a standard way as

$$
P=\int_{0}^{\tau}\left(I_{x}-I_{y}\right) d t / \int_{0}^{\tau}\left(I_{x}+I_{y}\right) d t
$$

where $I_{x . y}(t)$ is the intensity of lasing passing through a polarizer oriented along the axes associated with the extremes of the amplification factor $g(\psi)$ and $\tau$ denotes the pulse length or the sampling time. The statistical functions are derived using the Monte Carlo method, with the number of realizations being $10^{4}$.

\section{Results of numerical simulation}

Numerical simulation has been performed for a wide range of the parameters related to both edgeemitting (EEL) and VCSEL lasers. These results are qualitatively close. Therefore we present only the results obtained for the VCSEL based on GaAs heterostructure with the following set of averaged parameters: $g_{0}=40 \mathrm{~cm}^{-1}, \rho=8 \mathrm{~cm}^{-1}, L=3 \mu \mathrm{m}, R_{1}=R_{2}=0.99$, and $\varepsilon=10^{-6}$.

As testified by the results of numerical simulation, the variance of the distribution function for the input intensity is greater than that for the polarization degree. This difference is especially marked in the PS region when the following condition is met:

$$
\left|\frac{k_{x}-k_{y}}{k_{x}+k_{y}}\right|<10^{-3} .
$$

This is illustrated by Fig. 1 which presents, for a comparison, the bar charts of the output-radiation polarization degree and the intensity under the condition that the injection current is close to the PS point and the average polarization degree (or its value without fluctuations) is close to zero. However, the behaviours of distribution functions for the intensity and the polarization degree differ considerably when the pulse parameters vary in this region. In particular, with a fixed level of the variance $\xi_{\max }$, the characteristics of the intensity distribution function are practically
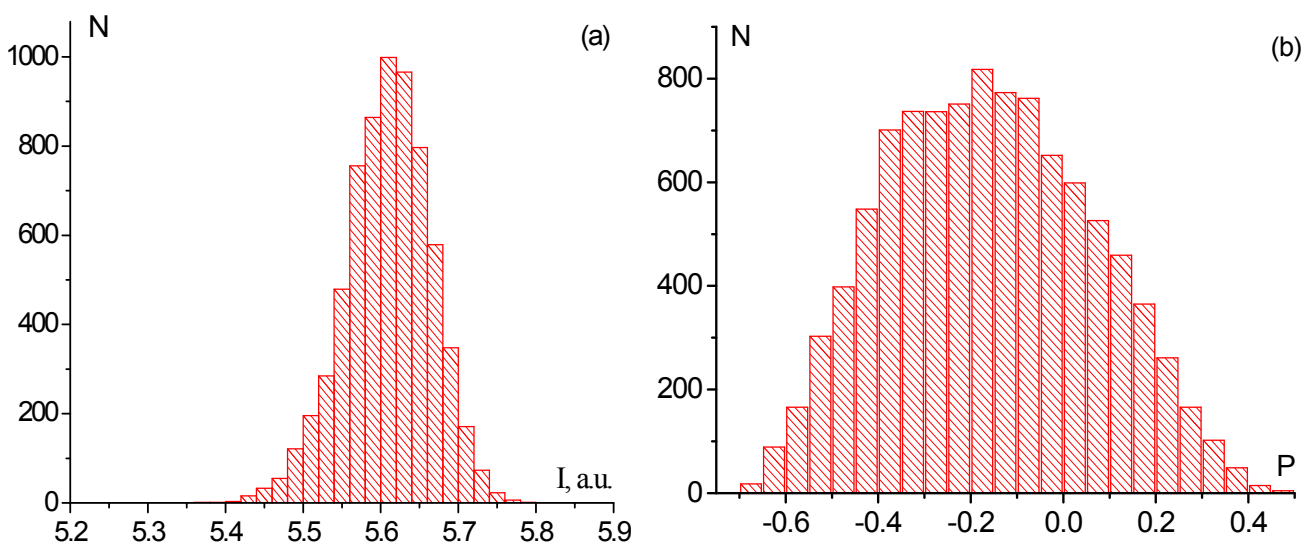

Fig. 1. Bar charts for the distributions of intensity (a) and polarization degree (b) of the output radiation: $\sigma=0.05, \tau=30 \mathrm{~ns}$, and $\left(k_{x}-k_{y}\right) /\left(k_{x}+k_{y}\right)=5 \times 10^{-4}$. 
independent of the pulse length $\tau$, whereas the variance of the polarization-degree distribution function increases proportionally to $\tau$. This may be explained on the basis of the results given in Ref. [8], where it has been demonstrated that the degree of polarization varies almost linearly over the trapezoid pulse. However, the shape of the distribution function changes with increasing variance: first the function simply 'spreads' (see Fig. 1a) and then (when the polarization degree range is limited) maxima are formed close to the limiting values \pm 1 . With further increase in the pulse length, these maxima become dominant (see Fig. 2a), indicating that, due to the fluctuations, the laser system approaches one of its states with limiting polarization degrees.
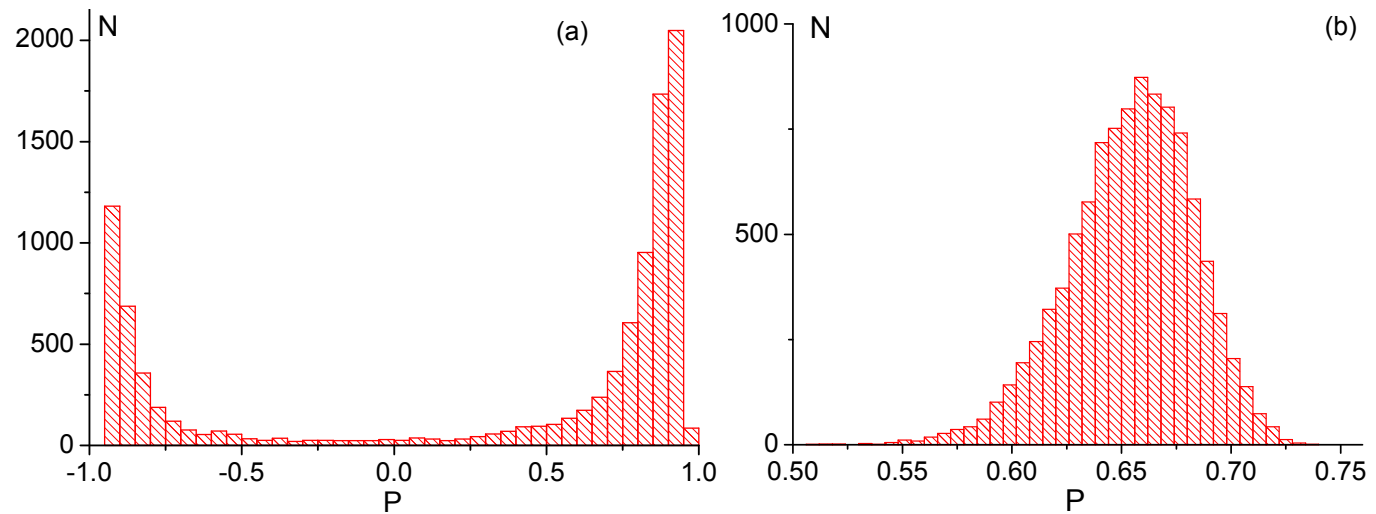

Fig. 2. Bar charts for the distributions of polarization degree of the output radiation: (a) $\sigma=0.05, \tau=80 \mathrm{~ns}$, and $\left(k_{x}-k_{y}\right) /\left(k_{x}+k_{y}\right)=5 \times 10^{-4} ;(\mathrm{b}) \sigma=0.05, \tau=30 \mathrm{~ns}$, and $\left(k_{x}-k_{y}\right) /\left(k_{x}+k_{y}\right)=2 \times 10^{-3}$.

A shift relative to the PS point (still in the region of PS) leads to a different pattern of transformation experienced by the distribution function for the polarization degree. 'Spreading' of the function is asymmetric, its maximum being slightly shifted towards one of the limiting values (see Fig. 2b). As the pulse length increases, the maximum of the distribution function shifts towards the region of the corresponding limiting value and the function acquires a long 'tail'. Its shape follows

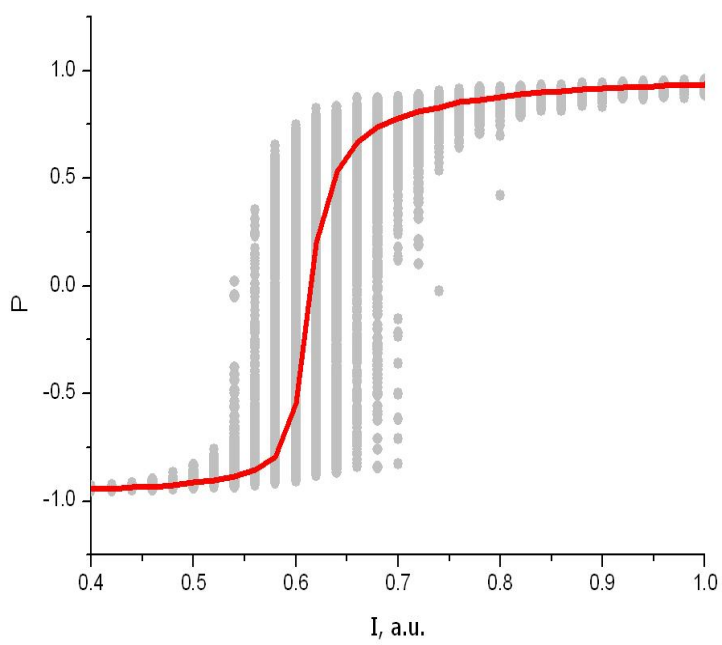

Fig. 3. Range of polarization degrees for the output radiation occurring under polarization switching: red line represents a switching curve in the absence of noise ( $\sigma=0.05$ and $\tau=50 \mathrm{~ns}$ ). that of the left (or right) part of the function given in Fig. 2a. Actually, this means that the most probable are the states with the limiting polarization degrees, which may be associated with the polarization modes. At the point of PS, the probabilities of transition to one of the limiting states are comparable and this fact may be interpreted such that the mode hopping process is developed. At the same time, the PS process has a statistical nature. Fig. 3 shows the region of possible values for the polarization degree and the curve that corresponds to the PS process with no fluctuations. 
Considering transformation dynamics of the distribution function for the output intensity and the polarization degree under the condition of fixed pulse length $\tau$, one can see that the shape of the intensity distribution function is slightly varying and its variance is proportional to $\tau$. Nonetheless, transformations of the polarization-degree distribution function reveal all of the features mentioned above: at a certain $\xi_{\max }$ value the probability of the event that the system is in one of the limiting states becomes prevalent. Note, however, that this $\xi_{\max }$ value is not strictly defined since the whole transformation process is smooth and has no singularities. Anyway, this value of $\xi_{\max }$ is inversely proportional to the pulse length $\tau$, in complete agreement with the model suggested in the studies [7, 8], i.e. the formation of polarization at a small orientational anisotropy of the amplification factor is rather slow process (the time of polarization formation is inversely proportional to $\left|k_{x}-k_{y} / k_{x}+k_{y}\right|<10^{-3}$ ), approaching the limiting values only in the case when the pulse length is sufficient for its completion. In fact this implies that, strictly speaking, the minimal pulse length associated with marked manifestations of the 'mode hopping' does not represent a characteristic of the laser system.

\section{Conclusion}

As demonstrated by the results of our numerical simulation within the scope of the model presented in Refs. [7, 8], the fluctuations in semiconductor lasers may lead to an abrupt (stepwise) change in the polarization state, which is usually referred to as the 'mode hopping'. Besides, development of this effect takes a finite time, in fairly good agreement with the experimental findings $[10,11]$. However, this time may be regarded as a characteristic of the laser system only if the noise is independent of the injection current. Even in the latter case, this parameter will be averaged and statistical in its character.

\section{References}

1. Panajotov K and Prati F, 2013. Polarization dynamics of VCSELs, in VCSELs. Springer Series in Optical Sciences. 166: 181-231.

2. Chang-Hasnain C J, Harbison J P, Hasnain G, Van Lehmen A C, Florez L T and Stoffel N G, 1991. Dynamic, polarization and transverse mode characteristics of vertical-cavity surfaceemitting lasers. IEEE J. Quantum. Electron. 24: 1402-1409.

3. Kawaguchi H, 1997. Bistable laser diodes and their applications: state of art. IEEE J. Select. Topics Quantum. Electron. 3: 1254-1270.

4. San Miguel M, Feng Q and Moloney J V, 1995. Light-polarization dynamics in surfaceemitting semiconductor lasers. Phys. Rev. A. 52: 1728-1739.

5. Verschaffelt G, Panajotov K, Albert J, Nagler B, Peeters M, Danckaert J, Veretennicoff I and Thienport H, 2001. Polarization switching in vertical-cavity surface-emitting lasers: from experimental observations to applications. Opto-Electron. Rev. 9: 257-268.

6. Tan Y, Zhang S, Ren C and Ren Z, 2009. Self-mixing interference effects in orthogonally polarized dual-frequency Nd:YAG lasers at different feedback levels. J. Phys. B. 42: 025401.

7. Jadan M, Burov L I, Gorbatsevich A S and Sokolov E S, 2009. Polarization switching in single-mode semiconductor laser. J. Appl. Spectrosc. 76: 678-684.

8. Jadan M, Burov L I, Gorbatsevich A S and Sokolov E S, 2010. Dynamics of polarization switching in single-mode injection semiconductor lasers. J. Appl. Spectrosc. 77: 65-72.

9. Willemsen M B, Khalid MUF, van Exter M P and Woerdman J P, 1999. Polarization 
switching of a vertical-cavity semiconductor laser as a Kramers hopping problem. Phys. Rev. Lett. 82: 4815-4818.

10. Nagler B, Peeters M, Albert J, Verschaffelt G, Panajotov K, Thienport H, Veretennicoff I, Danckaert J, Barbay S, Giacomelli G and Marin F, 2003. Polarization-mode hopping in single-mode vertical-cavity surface-emitting lasers: Theory and experiment. Phys. Rev. A. 68: 013813.

11. Sciamanna M, Masoller C, Abraham N B, Rogister F, Megret P and Blondel M, 2003. Different regimes of low-frequency fluctuations in vertical-cavity surface-emitting lasers. J. Opt. Soc. Amer. B. 90: 37-44.

12. Jadan M, Burov L I and Gorbatsevich A S, 2012. Point model for describing the polarization parameters of a single-mode semiconductor laser. J. Appl. Spectrosc. 79: 577-582.

M. Jadan 2014. Mode hopping and carrier density fluctuations in semiconductor lasers. Ukr.J.Phys.Opt. 15: 17 - 23.

Анотація. В роботі розглянуто вплив шуму на потужність $i$ поляризаційні характеристики напівпровідникових лазерів, в межах намої попередньої моделі, яка розглядає переполяризацію, як послідовний прочес трансформації поляризації. Показано, що, за певних умов, можна спостерігати поетапний перехід між станами з граничними значеннями ступеня поляризації, хоча вони мають статистичний характер. Цей прочес вимагає певного часу для свого розвитку, який не може розглядатися як параметр лазерної системи. 